\title{
Numerical Methods in Calculating Eigenvalues: Case Studies in Stability of Euler Columns
}

\author{
Hammad Jamil $^{1 *}$, Naki Tutuncu ${ }^{2}$ \\ ${ }^{1,2}$ Cukurova University, Department of Mechanical Engineering, 01330 Adana, TURKEY \\ *hammadjamil.nust@gmail.com
}

\begin{abstract}
A comparative analysis of well renowned "Shooting Method" with another numerical method "Complementary Functions Method" (CFM) is presented for calculating eigenvalue $(\lambda)$. Contrary to the shooting method hit and trial approach, CFM exploits the properties of linear ordinary differential equation (LODE). In the case of linear eigenvalue Boundary value Problem (BVP), CFM generates an algebraic equation system with one unknown " $\lambda$ " and, alone root finding method is sufficient to give required eigenvalue. However, the Shooting Method create a system of algebraic equations containing two unknowns " $\lambda$ " and "missing initial conditions", that demands an additional numerical technique along with root finding method. These radical differences between two approaches, sets the basis for this comparative investigation. As a case study in Linear Elastic Stability, different cases of Euler columns are investigated by finding eigenvalues for each case numerically, under both methods. Comparison is performed on the basis of results accuracy and cost effectiveness for both numerical techniques while solving linear stability problems.
\end{abstract}

Keywords: Shooting method, Complementary Functions Method (CFM), eigenvalue, linear ordinary differential equation (LODE), Linear Elastic Stability, Linear eigenvalue boundary value problem, Euler Columns.

\section{INTRODUCTION}

Solving eigenvalue boundary value problems is one of the basic requirements while dealing with mechanical stability cases. In such problems, non-availability of analytical or closed-form solutions leaves us only with numerical methods. Further, the function of used numerical methods is not limited to conversion of a BVP into IVP but also determining correct eigenvalues. This extension in task demands greater efficiency from the used numerical method.

Shooting method has been extensively used for solving the regular and Eigen value BVP due to its simple and direct approach. In general shooting method's main role is determining the missing initial conditions by suitable root finding numerical methods. In the case of eigenvalue BVP, Presence of an eigenvalue as an additional variable restricts the direct use of shooting method. D.J.Jone's [1] used an additional step to find eigenvalues by shooting method. Firstly use of the Least Square Method by incrementing the guessed $\lambda$ from 0 to 100,000 in steps of 20 is employed to get the traces of first few eigenvalues. Later it is followed by Newton's iteration as a root finding method. Even with an additional step, Shooting Method with its direct guessing approach required massive number of iterations to reach the correct eigenvalue. Xi Chen [2] presented a generalized approach with a mathematical proof of using the Shooting Method for an 
eigenvalue BVP. An equation derived from the Schrodinger equation is solved with the same guessing approach. In recent years use of Shooting Method for eigenvalue BVP arise from stability and free vibration problems can be seen commonly. Zhou \& Zheng [3] use the Shooting Method while doing thermal buckling analysis of an elastic rod with fixed ends. A set of algebraic equations is generated containing two unknown's " $\lambda$ " and "missing initial conditions" with a condition to satisfy boundary conditions. Here Newton-Raphson iteration is employed along with analytical continuation for " $\lambda$ " to find the root of above equations. Li [4] extended thermal buckling problem by including vibration. Here Shooting Method is used to find two eigenvalues by the same approached mentioned earlier. Increase in the number of unknowns means increase in the number of guesses that will further reduce the efficiency of the Shooting Method. Zhu [5] introduce a technique to improve the initial guessing during root finding in shooting method for calculating bifurcation points. A perturbation form of original problem is considered and then bisection method is used to find the perturbation points. In [6] and [7] Shooting Method is used for solving FGM discs buckling and free vibration cases. In light of the above discussion, effectiveness of Shooting Method with its hit and trail approach especially for solving stability problems comes under question.

In past, CFM has been used by many researchers to solve mechanical and applied mathematics problems. [8], [9] \& [10] CFM is used to solve both linear and non-linear BVP. [11] \& [12] applied CFM to solve different instability problems. [13] - [16] CFM is used for solving different free and forced vibration cases of composite beams and arches. In [17] CFM is employed to perform transient analysis of composite parabolic arches. [18] Uses CFM to study the effect of the Poisson ratio on stresses of heterogeneous pressure vessels. [19] Applied CFM while studying the effect of uniform magnetic field on pressurized FG cylindrical and spherical vessels. In the present paper application area of CFM method is extended by using it first time for solving Buckling stability problem and also comparing with Shooting Method. Ability of CFM that it removes the need of guessing initial conditions while solving BVP makes it an extremely robust method. Further, it gives solution by separately finding complementary and particular solutions. In the case of eigenvalue BVP, Complementary and particular solutions generates a single algebraic eq. containing $\lambda$ and solving it for root give us required eigenvalue.

\section{GOVERNING EQUATIONS}

Consider an axially loaded slender rod as an Euler Column of length " $L$ " with flexural rigidity of "D=EI" under the compressive force "P". Differential boundary equations and analytical expression of eigenvalue for the buckling of Euler Columns under various boundary conditions are given by [1]. X and Y represent axial coordinate and deflection of rod respectively. Where $\lambda=P / D$ and bending moment is given by $M_{0}$.

$$
\begin{aligned}
\frac{d Y}{d X} & =Q(X, \lambda, Y) \quad, \quad 0<X<1 \\
\mathrm{Y} & =\left\{\mathrm{y}_{1}, \mathrm{y}_{2}\right\}^{\mathrm{T}}
\end{aligned}
$$

Both ends hinged (H-H)

$$
\begin{array}{ll}
\mathrm{Q}=\left\{\mathrm{y}_{2},-\lambda^{2} \mathrm{y}_{1}\right\}^{\mathrm{T}} & \mathrm{y}_{1}(0)=\mathrm{y}_{1}(1)=0 \\
\lambda=\frac{n \pi}{L} &
\end{array}
$$

Both ends fixed (F-F)

$$
\begin{aligned}
& \mathrm{Q}=\left\{\mathrm{y}_{2},-\lambda^{2} \mathrm{y}_{1}+\frac{M_{0}}{D}\right\}^{\mathrm{T}} \quad \mathrm{y}_{1}(0)=\mathrm{y}_{2}(0)=\mathrm{y}_{1}(1)=\mathrm{y}_{2}(1)=0 \\
& \lambda=\frac{2 n \pi}{L}
\end{aligned}
$$


One end fixed and one end hinged $(\mathrm{F}-\mathrm{H})$

$$
\begin{aligned}
& \mathrm{Q}=\left\{\mathrm{y}_{2,}-\lambda^{2} \mathrm{y}_{1}+\frac{M_{0} X}{D L}\right\}^{\mathrm{T}} \quad \mathrm{y}_{1}(0)=\mathrm{y}_{1}(1)=\mathrm{y}_{2}(1)=0 \\
& \lambda=\frac{4.49 n}{L}
\end{aligned}
$$

One end fixed and one end free (F-F')

$$
\begin{aligned}
& \mathrm{Q}=\left\{\mathrm{y}_{2},-\lambda^{2}\left(\mathrm{y}_{1}+\delta\right)\right\}^{\mathrm{T}} \quad \mathrm{y}_{1}(0)=\mathrm{y}_{1}(1)=0 ; \mathrm{y}_{1}(1)=\delta \\
& \lambda=\frac{n \pi}{2 L}
\end{aligned}
$$

\section{NUMERICAL METHODS FORMULATION}

The shooting method and complementary function method are used to determine the eigenvalue for each case numerically.

\section{1. $\quad$ Shooting Method}

By considering missing initial conditions as a multivariable function of given boundary conditions; the boundary value problem for all cases are converted into initial value problems.

From equation (1) and equation (2) the initial value problem for $(\mathrm{H}-\mathrm{H})$ is given as

$$
(H-H) \quad\left\{\begin{array}{l}
\frac{d Y}{d X}=Q(X, \lambda, Y) \\
\mathrm{Y}(0)=\{0, \beta\}^{\mathrm{T}}
\end{array} \quad, \quad 0<X<1\right.
$$

Here $\beta$ is the guess value for the missing initial condition. For a certain value of $\beta$ and $\lambda$, denote the solution of equation (10) as $\mathrm{y}(X, \lambda, \beta)$ such that the value of $\beta$ and $\lambda$ satisfy the following algebraic equation

$$
(H-H) \quad(1 ; \lambda, \beta)=0
$$

Initial value problem Eq.10 is needed to be solve simultaneously with an Eq.11.An iterative scheme is set up by using RK4 method to integrate the Eq.(10) and secant method to find the $\operatorname{root} \beta$ of Eq.(11). Analytical continuation is employed on $\lambda$ by increasing it values on each step from an infinitesimal value. Similarly for other Euler Columns an iterative scheme is formulated.

From equation (1) and equation (4) the initial value problem for $(\mathrm{H}-\mathrm{H})$ is given as

$$
\begin{aligned}
& (F-F) \quad\left\{\begin{array}{c}
\frac{d Y}{d X}=Q(X, \lambda, Y) \\
Y(0)=\{\beta, 0\}^{\mathrm{T}}
\end{array}, \quad 0<X<1\right. \\
& (F-F) \quad(1 ; \lambda, \beta)=0
\end{aligned}
$$


From equation (1) and equation (6) the initial value problem for $(\mathrm{H}-\mathrm{H})$ is given as

$$
\begin{aligned}
& (F-H) \quad\left\{\begin{array}{l}
\frac{d Y}{d X}=Q(X, \lambda, Y) \\
\mathrm{Y}(0)=\{0, \beta\}^{\mathrm{T}}
\end{array}, \quad 0<X<1\right.
\end{aligned}
$$

From equation (1) and equation (8) the initial value problem for $(\mathrm{H}-\mathrm{H})$ is given as

$$
\begin{array}{ll}
\left(F-F^{\prime}\right) & \left\{\begin{array}{l}
\frac{d Y}{d X}=Q(X, \lambda, Y) \\
\mathrm{Y}(0)=\{0, \beta\}^{\mathrm{T}}
\end{array}, \quad 0<X<1\right.
\end{array}
$$

\subsection{Complementary Function Method}

Solution of $\mathrm{n}^{\text {th }}$ order Eigenvalue linear ordinary differential boundary value problem is composed of $\mathrm{a}_{\mathrm{m}}$ Complementary solutions with $\mathrm{c}_{\mathrm{m}}$ Constants and an expression for inhomogeneous solution $\mathrm{b}$.

$$
f(x, \lambda)=\sum_{i=1}^{m}\left\{a_{m}(x, \lambda) \cdot c_{m}\right\}+b(x) \quad g<x<h
$$

Considering a family of linearly independent initial conditions

$$
\begin{gathered}
\left\{\mathrm{a}_{1}(\mathrm{~g}), \ldots, \mathrm{a}_{\mathrm{m}}(\mathrm{g}), \mathrm{b}(\mathrm{g})\right\}^{T}=\left\{\begin{array}{llll}
1 . \ldots 0 & 0, \ldots, 0 \ldots .1 & 0,0 \ldots 0 & 0
\end{array}\right\}^{T} \\
\mathrm{a}_{1}(\mathrm{~g})=\left\{\mathrm{a}_{1}(\mathrm{~g})^{0}, \ldots, \mathrm{a}_{1}(\mathrm{~g})^{\mathrm{n}}\right\}, \mathrm{a}_{\mathrm{m}}(\mathrm{g})=\left\{\mathrm{a}_{\mathrm{m}}(\mathrm{g})^{0}, \ldots, \mathrm{a}_{\mathrm{m}}(\mathrm{g})^{\mathrm{n}}\right\}, \mathrm{b}(\mathrm{g})=\left\{\mathrm{b}(\mathrm{g})^{0}, \ldots ., \mathrm{b}(\mathrm{g})^{\mathrm{n}}\right\}
\end{gathered}
$$

RK4 is employed as a numerical integrator to find values of $a_{n}(h)$ and $b(h)$. On forcing boundary conditions $B_{m}$ generate a set of algebraic equations and solving them simultaneously gives $c_{n}$.

$$
\begin{gathered}
\mathrm{A}(\lambda) \cdot \mathrm{C}=0 \\
\mathrm{~A}(\lambda)=\left\{\mathrm{a}_{1}\left(\mathrm{~B}_{1}\right) \ldots \mathrm{a}_{\mathrm{m}}\left(\mathrm{B}_{1}\right), \mathrm{a}_{1}\left(\mathrm{~B}_{\mathrm{m}}\right) \ldots . \mathrm{a}_{\mathrm{m}}\left(\mathrm{B}_{\mathrm{m}}\right)\right\}^{T}, \quad C=\left\{\mathrm{c}_{1}, \ldots, \mathrm{c}_{\mathrm{m}}\right\}^{T}
\end{gathered}
$$

For a non-trivial solution $\mathrm{C} \neq 0$, hence

$$
\operatorname{Det}|A(\lambda)|=0
$$


CFM provides an algebraic equation containing only a single variable and direct application of secant method as root finding algorithm on equation (21) will generate eigenvalue. On following the above mentioned numerical scheme single variable algebraic equations are generated for all Columns cases.

From equation (1), equation (2) \& equation (18-20)

$$
(\boldsymbol{H}-\boldsymbol{H}) \quad \mathrm{A}(\lambda) \cdot \mathrm{C}=0
$$

$$
\mathrm{A}(\lambda)=\left\{1 \quad 0, \mathrm{a}_{1}(1) \mathrm{a}_{2}(1)\right\}^{T}, \quad C=\left\{\mathrm{c}_{1}, \mathrm{c}_{2}\right\}^{T}
$$

From equation (1), equation (4) \& equation (18-20)

$$
(\boldsymbol{F}-\boldsymbol{F}) \quad \mathrm{A}(\lambda) \cdot \mathrm{C}=0
$$

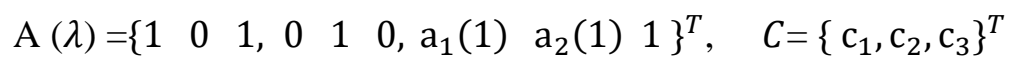

From equation (1), equation (6) \& equation (18-20)

$$
\begin{aligned}
& (\boldsymbol{F}-\boldsymbol{H}) \quad \mathrm{A}(\lambda) \cdot \mathrm{C}=0 \\
& \mathrm{~A}(\lambda)=\left\{1 \quad 0 \quad 0, \mathrm{a}_{1}^{1}(1) \quad \mathrm{a}_{2}^{1}(1) 1, \mathrm{a}_{1}(1) \mathrm{a}_{1}(1) 1\right\}^{T}, \quad C=\left\{\mathrm{c}_{1}, \mathrm{c}_{2}, \mathrm{c}_{3}\right\}^{T}
\end{aligned}
$$

From equation (1), equation (8) \& equation (18-20)

$$
\left(\boldsymbol{F}-\boldsymbol{F}^{\prime}\right) \quad \mathrm{A}(\lambda) . \mathrm{C}=\mathrm{S}
$$

$$
\mathrm{A}(\lambda)=\left\{\begin{array}{llllllll}
1 & 0 & 1,0 & 1 & 0, \mathrm{a}_{1}(1) \mathrm{a}_{2}(1) & 1
\end{array}\right\}^{T}, \quad C=\left\{\mathrm{c}_{1}, \mathrm{c}_{2}, \mathrm{c}_{3}\right\}^{T}, \quad S=\{0,0, \delta\}^{T}
$$

\section{NUMERICAL RESULTS \& DISCUSSION}

In order to establish a neutral medium for comparison; Secant method is applied as a root finding algorithm while Runge-kutta of $4^{\text {th }}$ order is used as numerical integrator for both methods. "Intel(r) Core(TM) i76500u CPU@2.50 GHz \& 2.60 GHz with 8GB Ram” operating system is used to perform iterations. Same initial guesses and tolerance values, iterations are carried out for finding first order $(n=1)$ eigenvalue. For simplicity, Consider $\mathrm{L}=\mathrm{D}=\mathrm{M}=\delta=1$. Accuracy of numerical results is given with respect to analytical results. Processing time for CFM is calculated for $10^{-6}$ accuracy and for Shooting Method it's restricted to $10^{-3}$ due to high number of iterations. 
Table 1. Numerical results comparison for $(\mathrm{H}-\mathrm{H})$

\begin{tabular}{ccc}
\hline & Shooting Method & CFM \\
\hline Step Size & 100 & 100 \\
No of Iterations & $3 \times 10^{4}$ & 6 \\
Accuracy & $10^{-3}$ & $10^{-6}$ \\
Processing Time & $28 \mathrm{~min}$ & $0.1 \mathrm{Sec}$ \\
\hline
\end{tabular}

Table 2. Numerical results comparison for (F-F)

\begin{tabular}{ccc}
\hline & Shooting Method & CFM \\
\hline Step Size & 100 & 100 \\
No of Iterations & $7 \times 10^{4}$ & 8 \\
Accuracy & $10^{-3}$ & $10^{-6}$ \\
Processing Time & $35 \mathrm{~min}$ & $0.2 \mathrm{Sec}$ \\
\hline
\end{tabular}

Solving first two cases H-H, F-F by shooting method gives another challenge in selection of known initial conditions. In cases of giving common initial conditions for both cases from equation (10)

$$
\mathrm{Y}(0)=\{0, \beta\}^{\mathrm{T}}
$$

Solution shape for F-F doesn't satisfy boundary conditions $\mathrm{y}_{2}(0)=\mathrm{y}_{2}(1)=0$. Unless, a set of uncommon initial conditions considering for F-F from equation (11)

$$
\mathrm{Y}(0)=\{\beta, 0\}^{\mathrm{T}}
$$

It signifies another drawback of using shooting method for stability problems.

Table 3. Numerical results comparison for (F-F)

\begin{tabular}{ccc}
\hline & Shooting Method & CFM \\
\hline Step Size & 100 & 100 \\
No of Iterations & $5 \times 10^{4}$ & 6 \\
Accuracy & $10^{-3}$ & $10^{-6}$ \\
Processing Time & $29 \mathrm{~min}$ & $0.2 \mathrm{Sec}$ \\
\hline
\end{tabular}


Table 1-3, depicts that shooting method needed a massive number of iterations along with huge processing time to find the missing initial conditions and eigenvalue with low accuracy of $10^{-3}$. Conversely, CFM requires fewer numbers of iterations and insignificant amount of processing time for calculating eigenvalue with double accuracy of $10^{-6}$.

Table 4. Numerical results comparison for $\left(\mathrm{F}-\mathrm{F}^{\prime}\right)$

\begin{tabular}{ccc}
\hline & Shooting Method & CFM \\
\hline Step Size & 100 & 100 \\
No of Iterations & $2 \times 10^{4}$ & $2 \times 10^{3}$ \\
Accuracy & $10^{-3}$ & $10^{-3}$ \\
Processing Time & $29 \mathrm{~min}$ & $11 \mathrm{~min}$ \\
\hline
\end{tabular}

In fixed-free case since given boundary conditions are not homogeneous, from equation(8)

$$
\mathrm{y}_{1}(0)=\mathrm{y}_{1}(1)=0 ; \mathrm{y}_{1}(1)=\delta
$$

As a result, here CFM gives a non-homogenous system of algebraic equations, from equation (10)

$$
\mathrm{A}(\lambda) \cdot \mathrm{C}=\mathrm{S}
$$

In order to solve the above non-homogenous system of algebraic equations, directly root finding method cannot be employed. Hence, equation (10) is solved iteratively for the successive values of $\lambda$ until both sides become equal and this process in literature is called as analytical expansion. It leads to increase in the number of iterations and processing time for CFM but still its performance is better than shooting method.

\section{CONCOLUSION}

A comparative investigation is carried out based upon the results accuracy and cost effectiveness, between the two numerical methods namely, Shooting Method and Complementary Function Method (CFM) respectively, for finding the eigenvalues in different cases of Euler Columns. Although Shooting Method is an effective direct approach for solving Ordinary boundary value problems, but in case of eigenvalue problems due to the introduction of another variable "Eigenvalue" $(\lambda)$, causes the significant increases in its number of iterations and processing time. On the other hand, CFM solves the same problem with double accuracy as of shooting method under fewer numbers of iterations and insignificant processing time. 


\section{References}

[1] D.J.Jones, "Use of a shooting method to compute eigenvalues of fourth- order two-point boundary value problems" ,Journal of Computational and Applied Mathematics, Volume 47, No. 3, PP. 395-400

[2] X.Chen, "The Shooting Method for Solving Eigenvalue Problems", Journal of Mathemtical Analysis and applications 203, 435]450 1996.

[3] S.Li, Y.H.Zhou \& X.Zheng (2002), "Thermal Postbuckling of a Heated Elastic Rod with Pinned-Fixed Ends", Journal of Thermal Stresses, 25:1, 45-56,

[4] S.-R. Li, "Vibration of Thermally Post-Buckled Orthotropic Circular Plates", Journal of Sound and vibration (2001), 248(2), 379\}386

[5] Y.ZHU, Y.J.Hu, C.J.Cheng, "Analysis of nonlinear stability and post-buckling for Euler-type beamcolumn Structure", Appl. Math. Mech. -Engl. Ed., 32(6), 719-728 (2011)

[6] Q.Li, "Shooting method for free vibration of FGM Reissner-Mindlin circular platesresting on elastic foundation in thermal environments", Journal of Vibroengineering 19(6) · October 2017

[7] L.S.Ma, Buckling of Functionally Graded Circular/Annular Plates Based on the First-Order Shear Deformation Plate Theory, Key Engineering Materials 261-263:609-614 · January 2004

[8] R.P.Agarwal, "On the method of complementary functions for nonlinear boundary-value problems", Journal of Optimization Theory and Applications volume 36, pages139-144(1982)

[9] A.Miele, "Method of particular solutions for linear, two-point boundary-value problems", Journal of Optimization Theory and Applications, July 1968, Volume 2, Issue 4, pp 260-273

[10]K.N.Murty, K.R.Prasad, Y.S.Rao, “On the Method of Complementary Functions for Linear and Nonlinear Two-Point Boundary Value Problems", Journal of Mathematical Analysis \& Applications, 167, 32-42 (1992)

[11]S.Yildirim,N.Tutuncu, "Effect of Magneto-Thermal Loads on the Rotational Instability of Heterogeneous Rotors", AIAA Journal, March 2019

[12]S.Yildirim,N.Tutuncu, "On the Inertio-Elastic Instability of Variable-Thickness Functionally-Graded Disks", Mechanics Research Communications, 91:1-6, May 2018

[13]Çalım, F. F. 2009. Free and forced vibrations of non-uniform composite beams. Composite Structures, 88, 413-423.

[14]Çalım, F. F. 2012. Forced vibration of curved beams on two-parameter elastic foundation. Applied Mathematical Modelling, 36, 964-973.

[15]Noori, A.R.Aslan, T. A. \& Temel, B. 2018b. An efficient approach for in-plane free and forced vibrations of axially functionally graded parabolic arches with nonuniform cross section. Composite Structures, 200, 701-710.

[16]Aslan, T, Temel, B, Noori, A. (2019). In-Plane Free Vibration Frequencies of Stepped Circular Beams. ALKÜ Fen Bilimleri Dergisi , 1 (1) , 1-7.

[17]Temel, B. \& Noori, A. R. 2019. Transient analysis of laminated composite parabolic arches of uniform thickness. Mechanics Based Design of Structures and Machines, 1-9. 
[18]Eker, M, Yarımpabuç, D , Çelebi, K . (2018). The Effect of the Poisson Ratio on Stresses of Heterogeneous Pressure Vessels. European Mechanical Science, 2 (2), 52-59. DOI: 10.26701/ems.360134.

[19]Yarımpabuç, D, TEMO, A. (2019) The Effect of Uniform Magnetic Field on Pressurized FG Cylindrical and Spherical Vessels, European Mechanical Science, 3 (4), 133-141. DOI: 10.26701/ems.585130 